This item was submitted to Loughborough's Research Repository by the author.

Items in Figshare are protected by copyright, with all rights reserved, unless otherwise indicated.

\title{
Making Cornish-Fisher fit for risk measurement
}

PLEASE CITE THE PUBLISHED VERSION

https://doi.org/10.21314/JOR.2019.408

\section{PUBLISHER}

Incisive Media @ Infopro Digital Risk (IP) Limited

VERSION

VoR (Version of Record)

\section{PUBLISHER STATEMENT}

This paper was published in the journal Journal of Risk and is available at https://doi.org/10.21314/JOR.2019.408.

\section{LICENCE}

CC BY-NC-ND 4.0

\section{REPOSITORY RECORD}

Lamb, John D., Maura E. Monville, and Kai-Hong Tee. 2019. "Making Cornish-fisher Fit for Risk Measurement”. figshare. https://hdl.handle.net/2134/33750. 


\title{
Research Paper
}

\section{Making Cornish-Fisher fit for risk measurement}

\author{
John D. Lamb, ${ }^{1}$ Maura E. Monville ${ }^{2}$ and Kai-Hong Tee ${ }^{3}$ \\ ${ }^{1}$ University of Aberdeen Business School, MacRobert Building, 581 King Street, \\ Aberdeen AB24 5UD, UK; email: j.d.lamb@abdn.ac.uk \\ ${ }^{2}$ University of Surrey, 388 Stag Hill, Guildford GU2 7XH, UK; \\ email: maura.monville@gmail.com \\ ${ }^{3}$ School of Business and Economics, Loughborough University, \\ Loughborough LE11 3TU, UK; email: k.tee@lboro.ac.uk
}

(Received September 21, 2017; revised and accepted June 12, 2018)

\section{ABSTRACT}

The truncated Cornish-Fisher inverse expansion is well known and has been used to approximate value-at-risk (VaR) and conditional value-at-risk (CVaR). The following are also known: the expansion is available only for a limited range of skewnesses and kurtoses, and the distribution approximation it gives is poor for larger values of skewness and kurtosis. We develop a computational method to find a unique, corrected Cornish-Fisher distribution efficiently for a wide range of skewnesses and kurtoses. We show that it has a unimodal density and a quantile function which is twice-continuously differentiable as a function of mean, variance, skewness and kurtosis. We extend the univariate distribution to a multivariate Cornish-Fisher distribution and show that it can be used together with estimation-error reduction methods to improve risk estimation. We show how to test the goodness-of-fit. We apply the Cornish-Fisher distribution to fit hedge-fund returns and estimate CVaR. We 
conclude that the Cornish-Fisher distribution is useful in estimating risk, especially in the multivariate case where we must deal with estimation error.

Keywords: conditional value-at-risk (CVaR); estimation error; goodness-of-fit; kurtosis; skewness.

\section{INTRODUCTION}

Consider how we might estimate the risk of investing in some combination of $n$ assets. We may choose one or more risk measures such as variance, value-at-risk (VaR), deviation measures or conditional value-at-risk (CVaR). Whatever we choose, we should deal with estimation error (Herold and Maurer 2006) whenever we estimate the risk measure. This is an error that arises from using $n>1$ sample statistics to estimate $n$ population statistics. Its effect is that the asset with the lowest measured risk has underestimated risk, while the asset with the highest measured risk has overestimated risk. This effect increases rapidly with $n$. It is made worse when we consider not just $n$ assets but combinations of them.

If variance were the only risk measure of interest, then the covariance-shrinkage method of Ledoit and Wolf (2004) might be enough. But usually we want risk measures like $\mathrm{CVaR}$ that change as skewnesses and kurtoses change. If we want to correct anything beyond mean and variance, or to compute estimates for combinations of assets, we cannot simply adjust the data, for example, by shifting and scaling. Rather, we want a multivariate estimate of the distribution.

Our choice of the Cornish-Fisher distribution may seem surprising. Although it has been used in risk estimation (Bali et al 2007; Liang and Park 2007), it has two obvious problems: it is valid only for a small range of skewnesses and kurtoses, and it is known to be inaccurate for estimating distributions not close to normal. Recently, Maillard (2012) suggested a correction. We improve this correction and develop a method to find the estimation quickly, with guaranteed convergence to a unique set of parameters for skewness and kurtosis in a region ( $\hat{R}$ of Figure 1$)$ large enough to fit most asset returns. We then extend it to a multivariate distribution.

We show that the corrected Cornish-Fisher distribution has many desirable properties. When fitting normal data, we get a normal distribution. Otherwise, the density function $f$ is smooth, unimodal (ie, it has only one maximum) and flat-tailed; that is, $f(x)>0$ for $x \in \mathbb{R}$. These are properties that we would usually expect in asset distributions. We also show that Cornish-Fisher VaR and CVaR risk estimates are twice-continuously differentiable functions of the coefficients of a linear combination of assets.

Formally, suppose that we have $n$ random variables with an unknown distribution function, although its shape must generalize the normal distribution. Suppose also 
that we know the mean $\mu_{i}$, variance $\sigma_{i}^{2}$, skewness $\kappa_{3 i}$ and (excess) kurtosis $\kappa_{4 i}$ of each variable. We seek a family $\mathcal{F}\left(\boldsymbol{p}_{i}\right)\left(\boldsymbol{p}_{i}=\left(\mu_{i}, \sigma_{i}, \kappa_{3 i}, \kappa_{4 i}\right), i=1, \ldots, n\right)$ of distributions to model the random variables, ideally one that we can use to fit a multivariate distribution of all of them. Typically, as in our example, $\kappa_{3 i}$ and $\kappa_{4 i}$ will differ substantially from zero.

We can use the normal family for $\mathcal{F}(\boldsymbol{p})$ if skewness and kurtosis are zero. If skewness is zero, we can use the elliptical family of distributions. If we ignore kurtosis and can limit skewness to $(-1,1)$, we can use the skew-normal distribution family. However, none of these conditions is likely in financial data. The Box-Cox power exponential (BCPE) distribution (Rigby and Stasinopoulos 2004) appears to be a plausible alternative to $\mathcal{F}(\boldsymbol{p})$ because it is a four-parameter family of distributions. However, its quantile function does not, in general, have a continuous derivative, and, if the distribution is not symmetric, it is truncated in one or other of its tails; hence our approach.

We find a multivariate distribution that fits a mean vector, covariance matrix, coskewness and co-kurtosis tensors. The use of higher co-cumulants is not uncommon in the literature. They have been used to evaluate investment fund performance (Moreno and Rodríguez 2009) and portfolio allocation (Hitaj et al 2012). Jondeau et al (2017) even developed a model to explain the drivers of co-skewness and cokurtosis, giving rise to a better explanation of stock returns. It is well known that higher co-cumulants are difficult to estimate accurately and often give rise to estimation error. Future research may explore how to deal with these issues more effectively. The multivariate Cornish-Fisher distribution gives us a practical way to estimate risk measures when we do not estimate skewness and kurtosis directly from the data.

Let $X$ be a random variable. Writing

$$
F_{X}(x)=\Phi(u)
$$

we can derive a power series expansion (Cornish and Fisher 1938; Hill and Davis 1968):

$$
x=\sum_{k=0}^{\infty} a_{k} u^{k} .
$$

We call it the Cornish-Fisher inverse expansion. Here, $a_{k}$ are polynomials in the cumulants of $X$. The expansion truncated to four terms is commonly used to approximate $F_{X}$, for example, to approximate conditional CVaR. We use it with a systematic correction. We write $C_{\boldsymbol{q}}^{2}$ for the set of functions of $\boldsymbol{q}$ that is twice-continuously differentiable over some region of interest. 
We use hedge-fund data and CVaR to illustrate the Cornish-Fisher distribution in risk estimation. For simplicity, we ignore time series effects in estimating cumulants in the data, although we could easily include them (see, for example, Gabrielsen et al 2015).

Section 2 shows how to correct the Cornish-Fisher expansion to get a distribution. Section 3 shows that it has desirable properties for risk measurement and discusses generalizations, the most important of which is a multivariate Cornish-Fisher distribution. Section 4 provides a practical example that shows how to fit the distribution and how to estimate CVaR. Supplements A-D (available online) give details of our derivation.

\section{THE CORNISH-FISHER EXPANSION}

Suppose $X$ has mean 0 and variance 1 . Then, (1.2) gives

$$
F_{X}^{-1}(u)=\sum_{k=0}^{\infty} a_{k}\left(\Phi^{-1}(u)\right)^{k}
$$

where each $a_{k}$ is a polynomial in the cumulants of $X$, and $\Phi$ is the standard normal distribution function. To use this in practice, we need to truncate the series. For the approximation to be increasing, the highest power of $k$ must be odd. In practice, the fourth-order $(k=3)$ approximation is used. This gives (see Cornish and Fisher 1938) $a_{2}=-a_{0}=s, a_{1}=1+5 s^{2}-3 k$ and $a_{3}=k-2 s^{2}$, with $s=\kappa_{3} / 6$ and $k=\kappa_{4} / 24$. So, the fourth-order expansion (see (1.2)) is

$$
x=\xi(u)=-s+\left(1+5 s^{2}-3 k\right) u+s u^{2}+\left(k-2 s^{2}\right) u^{3},
$$

giving the quantile function

$$
\tilde{F}^{-1}(u)=-s+\left(1+5 s^{2}-3 k\right) \Phi^{-1}(u)+s\left(\Phi^{-1}(u)\right)^{2}+\left(k-2 s^{2}\right)\left(\Phi^{-1}(u)\right)^{3} .
$$

Following Maillard (2012), we treat $s$ and $k$ as parameters because (2.4) and (2.5) show $\tilde{F}$ does not have skewness $s / 6$ or kurtosis $k / 24$ unless $s=k=0$. We write $q=s^{2}$ to simplify expressions containing only even powers of $s . \tilde{F}$ can only be a distribution function if $\xi$ is a strictly increasing function of $u$. It is straightforward to show (see Maillard 2012) that $\xi$ is strictly increasing in the region $R$, given by $q<3-2 \sqrt{2}$ and

$$
\frac{1+11 q-\sqrt{q^{2}-6 q+1}}{6}<k<\frac{1+11 q+\sqrt{q^{2}-6 q+1}}{6}
$$


The moments of the fourth-order Cornish-Fisher inverse expansion are given by Maillard (2012) (correcting a misprint and as functions of $s$ and $k$ ):

$$
\left.\begin{array}{rl}
\mu_{1}(s, k)=0 \\
\mu_{2}(s, k)=1 & +6 k^{2}-24 s^{2} k+25 s^{4}, \\
\mu_{3}(s, k)=6 & -76 s^{3}+510 s^{5}+36 s k-468 s^{3} k+108 s k^{2}, \\
\mu_{4}(s, k)=3 & +3348 k^{4}-28080 s^{2} k^{3}+1296 k^{3}-6048 s^{2} k^{2} \\
& +252 k^{2}-123720 s^{6} k+8136 s^{4} k-504 s^{2} k \\
& +24 k+64995 s^{8}-2400 s^{6}-42 s^{4}+88380 k^{2} s^{4} .
\end{array}\right\}
$$

The skewness and kurtosis are given by

$$
\hat{s}(s, k)=\frac{\mu_{3}(s, k)}{\left(\mu_{2}(s, k)\right)^{3 / 2}} \quad \text { and } \quad \hat{k}(s, k)=\frac{\mu_{4}(s, k)}{\left(\mu_{2}(s, k)\right)^{2}}-3 .
$$

Equations (2.4) and (2.5) implicitly define a function

$$
G(s, k)=(\hat{s}(s, k), \hat{k}(s, k))^{\mathrm{T}} .
$$

Maillard (2012) suggests $G$ might be invertible and tabulates some values. Figure 1 shows an empirical plot of $R$ (part (a)) and of $\hat{R}=\{G(s, k):(s, k) \in R\}$ (part (b)). If a distribution has skewness $\kappa_{3}$ and kurtosis $\kappa_{4}$, then we must have $\kappa_{4} \geqslant \kappa_{3}^{2}-2$. The gray regions in Figure 1 show the areas that are excluded by this inequality. The points and dashed lines are discussed in Section 4 . Both plots show lines on which $s$ or $k$ is constant.

$G$ is not globally invertible. We show in Supplement A online that we can write its Jacobian determinant as $|J(s, k)|=\mu_{2}^{9 / 2} S(q, k) / 144$, where $S(q, k)$ is a polynomial in $q$ and $k$ that has a root at approximately $(0,-0.139) \notin R$. However, $G$ remains useful if we can establish two things. First, $G$ has a unique inverse for $\left(\kappa_{3}, \kappa_{4}\right) \in \hat{R}$ so that $\tilde{F}^{-1}(u)$ is a twice-continuously differentiable function of $\kappa_{3}$ and $\kappa_{4}$. Second, there is an efficient method to obtain this inverse.

The method we use is essentially Newton's method, which is well known and efficient. Define

$$
J(s, k)=\left(\begin{array}{ll}
\frac{\partial \hat{s}}{\partial s} & \frac{\partial \hat{s}}{\partial k} \\
\frac{\partial \hat{k}}{\partial s} & \frac{\partial \hat{k}}{\partial k}
\end{array}\right),
$$

the Jacobian matrix of $G$. Supplement A online demonstrates that $G$ has a unique inverse for $\left(\kappa_{3}, \kappa_{4}\right) \in \hat{R}$ by showing $|J(s, k)|>0$ for $(s, k) \in R$. 
FIGURE $1 \quad R$ and $\hat{R}$.

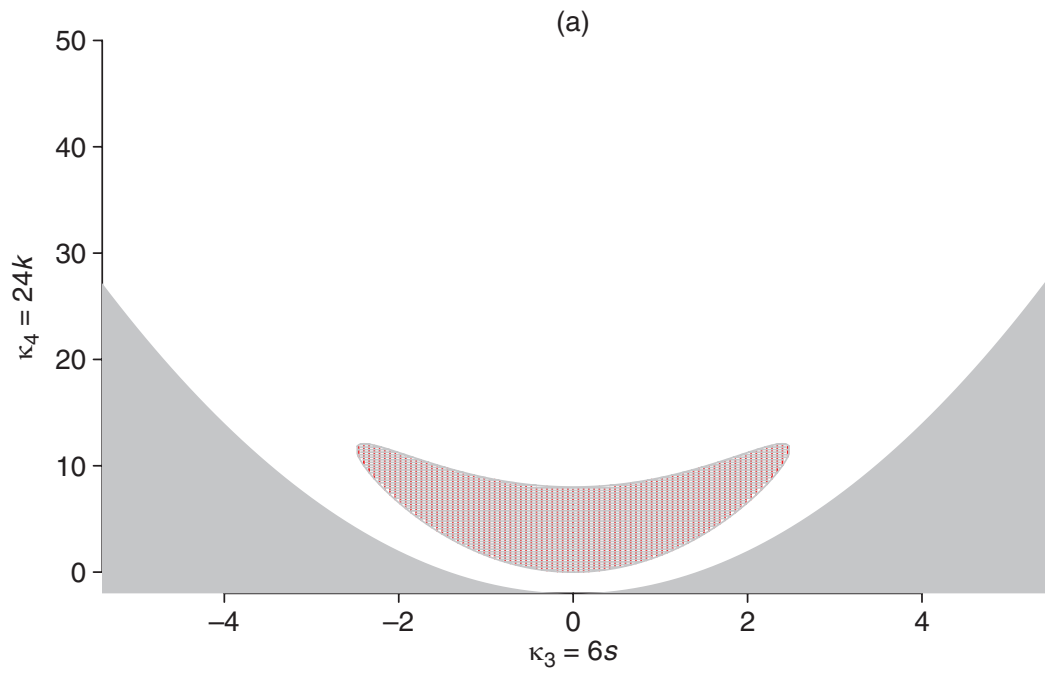

(b)

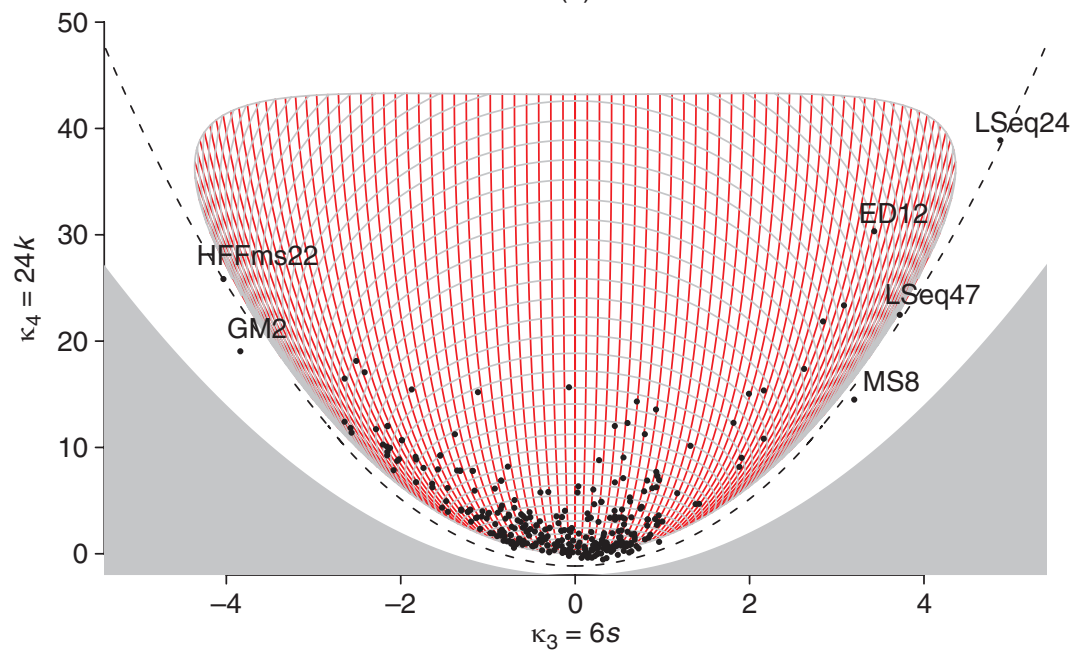

We solve $G(s, k)=\left(\kappa_{3}, \kappa_{4}\right)$ starting from $\left(s_{0}, k_{0}\right)$ by Newton's method. We compute $\left(s_{j}, k_{j}\right)$ iteratively using

$$
J\left(s_{j}, k_{j}\right)\left(\begin{array}{c}
\tilde{s}_{j} \\
\tilde{k}_{j}
\end{array}\right)=-\left(\begin{array}{c}
\hat{s}\left(s_{j}, k_{j}\right)-\kappa_{3} \\
\hat{k}\left(s_{j}, k_{j}\right)-\kappa_{4}
\end{array}\right), \quad\left(\begin{array}{l}
s_{j+1} \\
k_{j+1}
\end{array}\right)=\left(\begin{array}{c}
s_{j} \\
k_{j}
\end{array}\right)+\left(\begin{array}{c}
\tilde{s}_{j} \\
\tilde{k}_{j}
\end{array}\right) .
$$


For Newton's method to converge, we require $\hat{s}(s, k)$ and $\hat{k}(s, k)$ to be continuously differentiable, and $J(s, k)$ to be nonsingular wherever we evaluate it. We note that

$\mu_{2}(s, k)=1+6 k^{2}-24 q k+25 q^{2} \geqslant 1+6\left(k^{2}-4 q k+4 q^{2}\right) \geqslant 1+6(k-2 q)^{2} \geqslant 1$.

Hence $\hat{s}$ and $\hat{k}$ are twice-continuously differentiable. Since $G(0,0)=(0,0)$, we set $\left(s_{0}, k_{0}\right)=(0,0)$. By symmetry, we can assume $\kappa_{3} \geqslant 0$ and $s \geqslant 0$. Then, to ensure $J(s, k)>0$, we restrict Newton's method, replacing the equation on the right in (2.7) with

$$
\left(\begin{array}{c}
s_{j+1} \\
k_{j+1}
\end{array}\right)=\left(\begin{array}{c}
s_{j} \\
k_{j}
\end{array}\right)+\alpha\left(\begin{array}{c}
\tilde{s}_{j} \\
\tilde{k}_{j}
\end{array}\right),
$$

where we choose $\alpha \in(0,1]$ so that

- $\left(s_{j+1}, k_{j+1}\right)$ is in a convex subset containing the right-hand side of $R$ (see Figure 1) and

- the solution is improving.

Supplement A online explains this in more detail.

In practice, most nonlinear optimizers should be able to invert $G$, even without explicit derivatives, provided $s$ and $k$ are constrained to lie in a convex subset of $R$. Our method is merely efficient and allows us to invert $G$ for points very close to the dashed line in Figure 1(b), where the Jacobian is singular. The final paragraph of Section 4.2 explains this line in more detail.

Suppose that we want a distribution with mean $\mu$, variance $\sigma^{2}$, skewness $\kappa_{3}$ and kurtosis $\kappa_{4}$. Suppose also that $\mu$ and $\sigma$ are finite and $\left(\kappa_{3}, \kappa_{4}\right) \in \hat{R}$. Then, we can use Newton's method to evaluate $G^{-1}\left(\kappa_{3}, \kappa_{4}\right)$, giving us values for $s$ and $k$ and hence $a_{0}, a_{1}, a_{2}$ and $a_{3}$. Define

$$
F^{-1}(u ; \boldsymbol{p})=\mu+\sigma \mu_{2}^{-1 / 2} \sum_{j=0}^{3} a_{j}\left(\Phi^{-1}(u)\right)^{j}
$$

where $\mu_{2}$ is given by (2.4). $\tilde{F}$, defined by (2.2), has mean 0 , variance $\mu_{2}$, skewness $\kappa_{3}$ and kurtosis $\kappa_{4}$. Skewness and kurtosis are invariant under scaling and shifting. So, $F$ has mean $\mu$, variance $\sigma^{2}$, skewness $\kappa_{3}$ and kurtosis $\kappa_{4}$. We call it the CornishFisher distribution with parameters $\mu, \sigma, \kappa_{3}$ and $\kappa_{4}$. Thus, (2.9) defines a family of distributions for finite $\mu$, finite $\sigma>0$ and $\left(\kappa_{3}, \kappa_{4}\right) \in \hat{R}$. We write $Y \sim \mathcal{F}(p)$ to indicate $Y$ is a distribution in this family. For reasons that become clearer at the end of Section 3 and in Section 4, we occasionally abuse notation by referring to $F$ as a Cornish-Fisher distribution when $\left(\kappa_{3}, \kappa_{4}\right) \notin \hat{R}$. 
The Cornish-Fisher distribution that approximates a distribution by putting $s=$ $\kappa_{3} / 6$ and $k=\kappa_{4} / 24$ is widely used (Bali et al 2007; Liang and Park 2007). We call it the uncorrected Cornish-Fisher distribution and show (in Section 4) that it is sometimes a poor approximation.

\section{THE CORNISH-FISHER DISTRIBUTION AND A MULTIVARIATE GENERALIZATION}

We will now demonstrate some properties of the Cornish-Fisher distribution and a multivariate expansion.

If we wish to estimate risk by fitting a distribution to data, then we should be concerned about some of the properties of the distribution, particularly those of the left tail. We want $\operatorname{VaR}_{\alpha}$ and $\mathrm{CVaR}_{\alpha}$ to be well behaved. Usually, this means that we want them to be strictly increasing as functions of $\alpha$; that is, as $\alpha \rightarrow 0$ there is no point at which risk becomes zero and no range over which it is not decreasing. Consequently, we want a smooth distribution with a density that does not become zero at some point in the left tail, as, for example, the lognormal and gamma densities do.

Proposition 3.1 shows that the Cornish-Fisher distribution has these properties and consequently makes sense when estimating tail risk. We end Section 3.1 by showing that, even if we relax the region on which the Cornish-Fisher distribution is defined, $\mathrm{CVaR}_{\alpha}$ often remains well behaved for not-too-small $\alpha$.

In the multivariate case, we may wish to estimate the risk of some convex combination

$$
\sum_{i=1}^{n} \lambda_{i} X_{i}
$$

of assets $X_{1}, \ldots, X_{n}$ satisfying $\sum_{i=1}^{n} \lambda_{i}=1$ and $\lambda_{i} \geqslant 0$ for $i=1, \ldots, n$. Again, we want the risk measure to change smoothly as we change $\lambda=\left(\lambda_{1}, \ldots, \lambda_{n}\right)^{\mathrm{T}}$. Proposition 3.2 shows that this will happen provided we choose a smooth risk measure such as VaR or CVaR, and provided skewness and kurtosis also change smoothly with $\lambda$. They do so for the multivariate distribution of Section 3.2, which preserves the covariance matrix and co-cumulant tensors.

\subsection{Properties of the Cornish-Fisher distribution}

Suppose $Y \sim \mathcal{F}(\boldsymbol{p})$. Equations (2.1) and (2.9) give

$$
F^{-1}(u ; \boldsymbol{p})=\mu+\sigma \mu_{2}^{-1 / 2} \xi\left(\Phi^{-1}(u)\right)
$$


We can rearrange (3.1) to get the distribution function:

$$
F(x)=u=\Phi\left(\xi^{-1}\left(\mu_{2}^{1 / 2} \frac{x-\mu}{\sigma}\right)\right) .
$$

In the R-package in the supplementary material (available online), we use Newton's method to evaluate $\xi^{-1}$ rather than the formula for the roots of a cubic. This is because $\xi^{\prime}>0$ guarantees that the root is unique and that Newton's method converges.

The following two propositions are proved in Supplement B online. Note that the density function without the $\mu_{2}^{1 / 2}$ correction is given in Maillard (2013).

PROPOSITION 3.1 Let $Y \sim \mathcal{F}(\boldsymbol{p})$. The density function of $Y$ is

$$
f(x)=\frac{\mu_{2}^{1 / 2} \phi(v)}{\sigma \xi^{\prime}(v)}
$$

with $v=\xi^{-1}\left(\mu_{2}^{1 / 2}(x-\mu) / \sigma\right)$. The distribution function is smooth, and $f(x)$ is unimodal and satisfies $f(x)>0$ for $x \in \mathbb{R}$.

Note that smoothness and $f(x)>0$ are more important for risk estimation. However, unimodality increases our confidence: when we estimate risk through a distribution function, we want that function to match the properties of the true distribution as closely as possible.

Proposition $3.2 \quad F^{-1}$ is twice-continuously differentiable with respect to $\boldsymbol{p}=$ $\left(\mu, \sigma, \kappa_{3}, \kappa_{4}\right)$.

We can use the Cornish-Fisher distribution to estimate the $\alpha$ quantile, $F^{-1}(\alpha)$, and the (lower) $\alpha$ tail mean,

$$
\operatorname{TM}(X ; \alpha)=\mathbb{E}\left[X: X \leqslant F^{-1}(\alpha)\right],
$$

of $X$. These are often seen in finance as value-at-risk, $\operatorname{VaR}(X ; \alpha)=-F^{-1}(\alpha)$ (Tee 2009), and conditional-value-at-risk, $\operatorname{CVaR}(X ; \alpha)=-\operatorname{TM}(X ; \alpha)$. Acerbi (2002) shows that

$$
\operatorname{TM}(X ; \alpha)=\frac{1}{\alpha} \int_{0}^{\alpha} F^{-1}(u) \mathrm{d} u .
$$

Put

$$
\tau_{r}(\alpha)=\frac{1}{\alpha \sqrt{2 \pi}} \int_{-\infty}^{\Phi^{-1}(\alpha)} z^{r} \exp \left(-\frac{1}{2} z^{2}\right) \mathrm{d} z
$$


Then, if $Y$ has a Cornish-Fisher distribution approximating $X$,

$$
\begin{aligned}
\operatorname{TM}(Y ; \alpha) & =\frac{1}{\alpha} \int_{0}^{\alpha}\left(\mu+\sigma \mu_{2}^{-1 / 2}\left(\sum_{r=0}^{3} a_{r}\left(\Phi^{-1}(u)\right)^{r}\right)\right) \mathrm{d} u \\
& =\mu+\sigma \mu_{2}^{-1 / 2} \sum_{r=0}^{3} a_{r} \frac{1}{\alpha} \int_{0}^{\alpha}\left(\Phi^{-1}(u)\right)^{r} \mathrm{~d} u \\
& =\mu+\sigma \mu_{2}^{-1 / 2} \sum_{r=0}^{3} a_{r} \frac{1}{\alpha \sqrt{2 \pi}} \int_{-\infty}^{\Phi^{-1}(\alpha)} z^{r} \exp \left(-\frac{1}{2} z^{2}\right) \mathrm{d} z \\
& =\mu+\sigma \mu_{2}^{-1 / 2} \sum_{r=0}^{3} a_{r} \tau_{r}(\alpha) .
\end{aligned}
$$

This last expression is a twice-continuously differentiable function of $\boldsymbol{p}$, and so, by Proposition 3.2, VaR, TM and CVaR are also in $C_{\boldsymbol{p}}^{2}$. To use this result in practice, we need expressions to compute the partial derivatives of $F^{-1}$. Supplement $\mathrm{C}$ online derives such expressions.

The tail means and quantiles are only defined if $\xi(u)$ is strictly increasing on $\mathbb{R}$. Suppose, however, that $\xi(u)$ is increasing on $\left(u_{1}, u_{2}\right)$ and that $\alpha \in\left(\alpha_{1}, \alpha_{2}\right)$ with $\alpha_{1}=\Phi\left(u_{1}\right)$ and $\alpha_{2}=\Phi\left(u_{2}\right)$. Then, both $F^{-1}(Y ; \alpha)$ and $\operatorname{TM}(Y ; \alpha)$ are sensibly defined and are the quantile and tail mean of a distribution given by

$$
F^{\prime}(x)= \begin{cases}F_{1}(x), & x \leqslant x_{1}, \\ F(x), & x_{1}<x<x_{2}, \\ F_{2}(x), & x \geqslant x_{2},\end{cases}
$$

for $x_{1}=F^{-1}\left(u_{1}\right)$ and $x_{2}=F^{-1}\left(u_{2}\right)$ if we can find strictly increasing continuous functions $F_{1}$ and $F_{2}$ satisfying $F_{1}\left(x_{1}\right)=\alpha_{1}, F_{2}\left(x_{2}\right)=\alpha_{2}$, and

$$
\begin{aligned}
\frac{1}{\alpha_{1}} \int_{0}^{\alpha_{1}} F_{1}^{-1}(u) \mathrm{d} u & =\mu+\sigma \mu_{2}^{-1 / 2} \sum_{r=0}^{3} a_{r} \tau_{r}\left(\alpha_{1}\right), \\
\frac{1}{1-\alpha_{2}} \int_{\alpha_{2}}^{1} F_{2}^{-1}(u) \mathrm{d} u & =\mu+\sigma \mu_{2}^{-1 / 2} \sum_{r=0}^{3} a_{r} \tau_{r}\left(1-\alpha_{2}\right) .
\end{aligned}
$$

Such functions are easy to find. For example, $F_{1}^{-1}(u)=m u+x_{1}-m \alpha_{1}$ satisfies $F_{1}\left(x_{1}\right)=\alpha$ and

$$
\frac{1}{\alpha_{1}} \int_{0}^{\alpha_{1}} F^{-1}(u) \mathrm{d} u=x_{1}-\frac{1}{2} m \alpha_{1},
$$

and it is straightforward to show that

$$
\mu+\sigma \mu_{2}^{-1 / 2} \sum_{r=0}^{3} a_{r} \tau_{r}(\alpha)<x_{1} .
$$


So, if we choose $m$ satisfying (3.4), then $m>0$, and so $F_{1}$ is, as required, strictly increasing. It follows that if $\left(\kappa_{3}, \kappa_{4}\right) \notin \hat{R}$ but $\alpha \in\left(\alpha_{1}, \alpha_{2}\right)$, we can still use the Cornish-Fisher distribution to estimate quantiles and tail means. We return to this issue in Section 4.

\subsection{Generalizations and the multivariate Cornish-Fisher distribution}

We have established that the Cornish-Fisher distribution has very desirable properties and a region of validity that is much larger than we might have naively expected. We now consider generalizations.

Hill and Davis (1968) generalize Cornish-Fisher expansions to use nonnormal distributions. So, we might ask what happens if we replace $\Phi^{-1}$ in (2.9) with a nonnormal quantile function. We have tried this and found that if we replace $\Phi^{-1}$ with the quantile function of a beta, gamma or lognormal distribution, then $F$ fails to be a distribution function except in degenerate cases. The log of a Cornish-Fisher distribution is usually well defined. However, we find, in contrast with Proposition 3.1, that it is only unimodal in the most degenerate case. So, it has few practical uses.

In contrast, a multivariate Cornish-Fisher distribution is practically useful. We want to estimate the distribution of a vector $\left(X_{1}, \ldots, X_{n}\right)$ so that we may estimate risk measures of not only individual assets but also convex combinations of them; that is, of portfolios. We can write each portfolio as

$$
X=\sum_{j=1}^{n} \lambda_{j} X_{j}
$$

for $\lambda_{1}, \ldots, \lambda_{n} \geqslant 0$, satisfying $\lambda_{1}+\cdots+\lambda_{n}=1$.

One way to estimate a multivariate Cornish-Fisher distribution is to use data. If $r_{j t}$ is the $t$ th of $T$ observations from $X_{j}$, then

$$
r_{t}=\sum_{j=1}^{n} \lambda_{j} r_{j t}, \quad t=1, \ldots, T,
$$

is a vector of observations of the portfolio, with cumulants that we can use to estimate a Cornish-Fisher distribution, as before.

We generalize this. For various reasons, such as reducing estimation risk or accounting for time series effects, we may not wish to estimate the cumulants from the data directly but instead from tensors of means, covariance, co-skewnesses and co-kurtoses (Hitaj et al 2012; Moreno and Rodríguez 2009). 
Write $\mu_{1}, \ldots, \mu_{n}$ and $\sigma_{1}^{2}, \ldots, \sigma_{n}^{2}$ for the means and variances of $X_{1}, \ldots, X_{n}$. Then, co-skewness is defined as

$$
S_{i j k}=\frac{\mathbb{E}\left[\left(X_{i}-\mu_{i}\right)\left(X_{j}-\mu_{j}\right)\left(X_{k}-\mu_{k}\right)\right]}{\sigma_{i} \sigma_{j} \sigma_{k}},
$$

where $i, j$ and $k$ need not be distinct. $S_{i i i}$ is the skewness of $X_{i}$. Similarly, the co-kurtosis is

$$
K_{i j k l}=\frac{\mathbb{E}\left[\left(X_{i}-\mu_{i}\right)\left(X_{j}-\mu_{j}\right)\left(X_{k}-\mu_{k}\right)\left(X_{l}-\mu_{l}\right)\right]}{\sigma_{i} \sigma_{j} \sigma_{k} \sigma_{l}}
$$

and $K_{i i i i}$ is the kurtosis of $X_{i}$.

Suppose we have an estimate $s_{i j}$ of $\operatorname{cov}\left(X_{i}, X_{j}\right)$ (variance if $i=j$ ) for $i, j=$ $1, \ldots, n$. Then, we estimate the variance of the portfolio $X$ as

$$
s_{X}=\sum_{i=1}^{n} \sum_{j=1}^{n} \lambda_{i} \lambda_{j} s_{i j}
$$

Similarly, we estimate the skewness as

$$
S_{X}=\frac{1}{s_{X}^{3}} \sum_{i=1}^{n} \sum_{j=1}^{n} \sum_{k=1}^{n} \lambda_{i} \lambda_{j} \lambda_{k} S_{i j k}
$$

and the kurtosis as

$$
K_{X}=\frac{1}{s_{X}^{4}} \sum_{i=1}^{n} \sum_{j=1}^{n} \sum_{k=1}^{n} \sum_{l=1}^{n} \lambda_{i} \lambda_{j} \lambda_{k} \lambda_{l} K_{i j k l} .
$$

Thus, any estimate of the four co-cumulant tensors allows us to construct a multivariate Cornish-Fisher distribution.

Section 1 summarizes the univariate alternatives to the Cornish-Fisher distribution. We know of no general distribution defined by mean, covariance, co-skewness and co-kurtosis parameters that does not generalize one of these. The multivariate Cornish-Fisher distribution has the same advantages over multivariate alternatives as the univariate Cornish-Fisher distribution. Its weakness is that it is not well defined for the rare cases where it does not fit (see Section 4).

Note that the multivariate Cornish-Fisher distribution can be used whether we estimate the parameters directly from the data or use, for example, some time series model. It is especially helpful when the parameter estimates are not the same as those of the data, so that we have no empirical distribution from which to estimate risk measures. 
TABLE 1 Investment fund types.

\begin{tabular}{|c|c|c|c|}
\hline Prefix & Type & Prefix & Type \\
\hline HFFeq & $\begin{array}{l}\text { Hedge funds of funds } \\
\text { for equity }\end{array}$ & MS & Multistrategy \\
\hline HFFms & $\begin{array}{l}\text { Hedge funds of funds } \\
\text { for multistrategy }\end{array}$ & SF & Systematic futures \\
\hline HFFmasy & $\begin{array}{l}\text { Hedge funds of funds } \\
\text { for macrosystematic }\end{array}$ & GM & Global macro \\
\hline LSeq & $\begin{array}{l}\text { Long-short equity } \\
\text { strategy }\end{array}$ & ED & Event-driven strategy \\
\hline
\end{tabular}

\section{AN APPLICATION TO INVESTMENT FUND DISTRIBUTIONS}

We now investigate the practical applicability of the Cornish-Fisher distribution. We do this in four ways. First, we compare the Cornish-Fisher distribution with the uncorrected version to get a broad picture of how much the difference matters. We do this by simulating both distributions using intended parameters and comparing these with observed parameters. Second, we investigate how well a large data set fits the Cornish-Fisher distribution. We do this using goodness-of-fit tests and comparing our results with the uncorrected distribution. We also test the fit of the normal distribution to show that the data cannot plausibly have zero skewness and kurtosis. Third, we investigate the value of using the Cornish-Fisher distribution with a risk estimate (CVaR). Estimating risk in this manner is mostly valuable when we are using a model that does not allow us to estimate risk directly from the data. Here, however, we mainly use cases where an empirical CVaR estimate is also possible, so that we can directly compare two methods. Finally, we investigate the extent to which we can fit the multivariate Cornish-Fisher distribution to the entire data set.

To illustrate the Cornish-Fisher distribution, we consider the monthly returns of 339 investment funds from January 2000 to December 2012. We choose this data because investment returns often plausibly have a unimodal density that is too skewed, platykurtic or leptokurtic to be normal. This data originates from hedge funds. We collected monthly returns for the various strategies listed in Table 1. The data comes from Morningstar (2014), which describes the strategies in detail.

The points in Figure 1(b) show the skewness and kurtosis (estimated by $k$ statistics) for each investment except GM15, HFFms9 and SF49, which have skewness $5.672,-8.099$ and 6.26 (beyond the range of the chart). The prefix on the labeled fund shows its type. We choose a range of types to ensure a range of values of skewness and kurtosis. Table 1 shows the prefixes and fund types. 
FIGURE 2 Comparison of Cornish-Fisher (black) and uncorrected (gray) distributions.

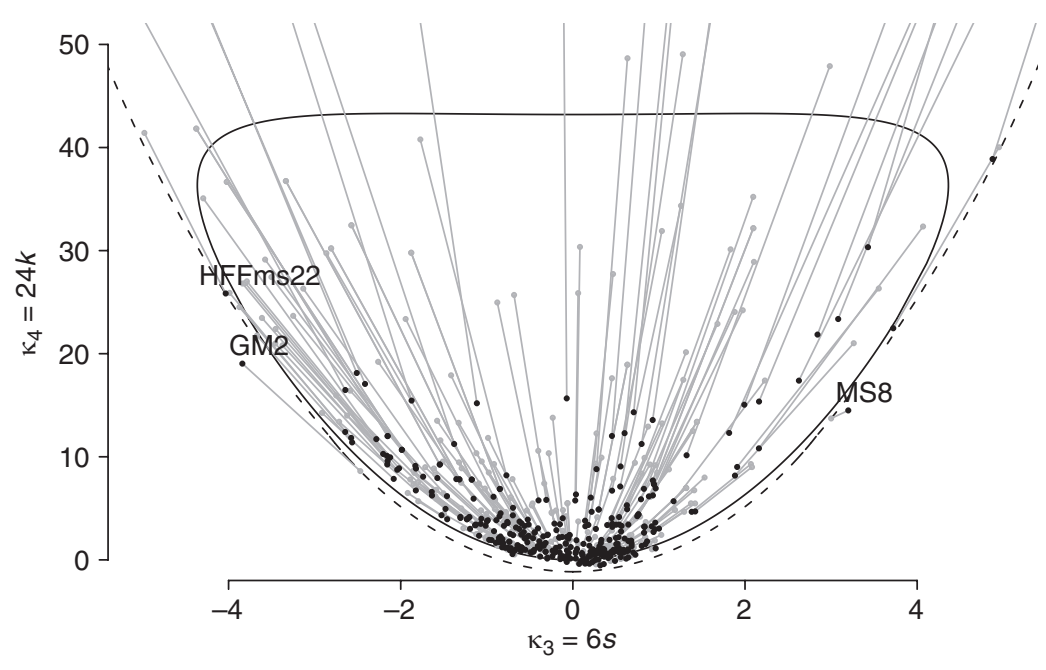

\subsection{Comparison with the uncorrected Cornish-Fisher distribution}

We first compare the Cornish-Fisher distribution with the uncorrected CornishFisher distribution. Figure 2 shows the skewnesses and kurtoses of the funds of Figure 1 as black points. The gray points show the skewnesses and kurtoses of the uncorrected Cornish-Fisher distributions; we have joined corresponding points with a gray line to show the effect of not using the correction. The Cornish-Fisher distribution has the same skewness and kurtosis as the data except for GM2, HFFms22, MS8 and the three points we noted above that are beyond the chart's range.

The solid and dashed black lines are described in Section 2.

We omit comparisons of mean and variance, because both distributions give the same values. Note that the uncorrected Cornish-Fisher distribution still uses the $\mu_{2}^{-1 / 2}$ correction of (3.1), although we are not aware of even this correction being used in the published literature.

Although the uncorrected distribution is reasonable for many of the funds, a substantial proportion of these funds have greatly exaggerated skewness and kurtosis that sometimes falls outside of the region where the distribution is valid.

\subsection{Fitting the Cornish-Fisher distribution}

We fit Cornish-Fisher distributions for as many of the funds as we can using the R-package in the supplementary material (available online). We do this even if 
FIGURE 3 Anderson-Darling $p$-values for Cornish-Fisher and normal distributions.

(a)

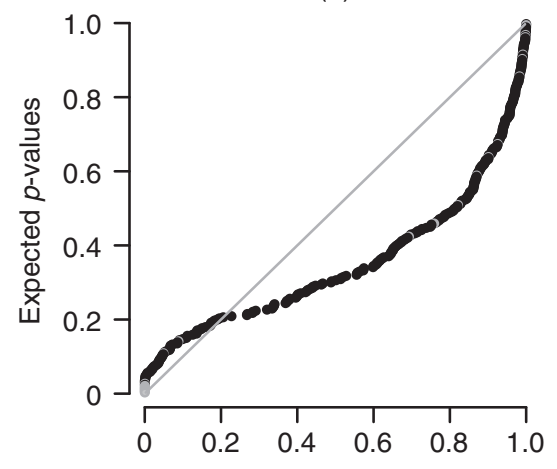

Observed Cornish-Fisher $p$-values (b)

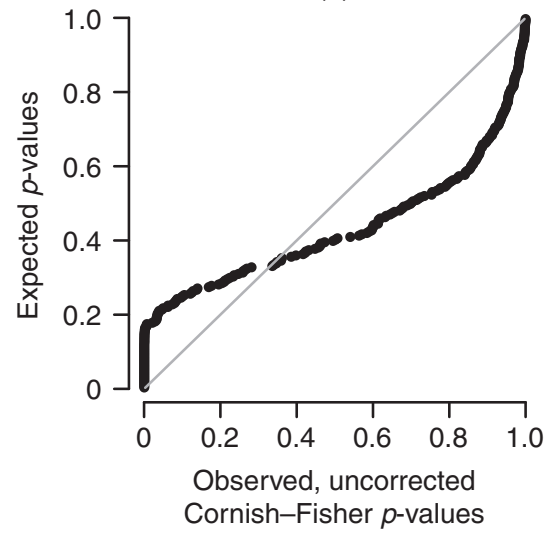

(c)

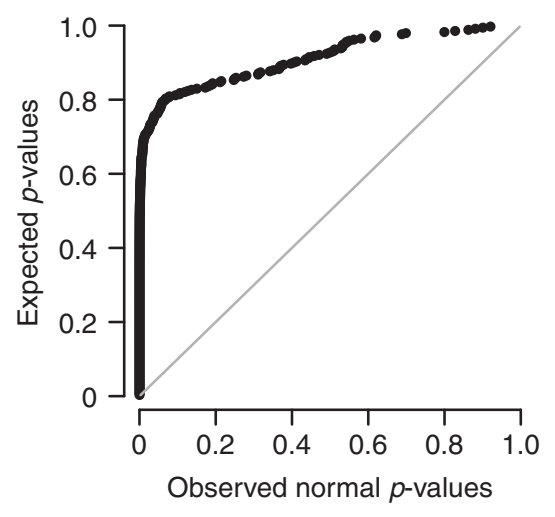

$\left(\kappa_{3}, \kappa_{4}\right) \notin \hat{R}$, and we compute a bootstrap Anderson-Darling (Cheng 2006) test statistic whenever we can. We fail to fit Cornish-Fisher distributions for seven funds: GM2, GM15, HFFms9, HFFms22, LSeq24, MS8 and SF49. All are strongly skewed. GM4, LSeq22 and thirteen SF funds also have $\left(\kappa_{3}, \kappa_{4}\right) \notin \hat{R}$. We find Cornish-Fisher distributions for them when we relax the requirement that the distribution function is defined in its tails. We tried using a Jarque-Bera in place of the Anderson-Darling test and found very similar results.

If we assume all fund returns have Cornish-Fisher distributions with known parameters and are independent, then the $p$-values from the Anderson-Darling tests should be uniformly distributed on $[0,1]$. Figure 3(a) shows the observed and 
expected $p$-values under this assumption. Note that Figure 3 does not show $p-p$ plots. We are not comparing the observed and expected values of a single distribution; rather, we are comparing the $p$-values of Anderson-Darling tests of the fits of three families of distributions with asset returns under the null hypothesis that each asset fits the family with some set of parameter values. This comparison is made under the assumption that all the null hypotheses are true for the given family. Under this assumption, we expect to observe $p$-values that are uniformly distributed on $[0,1]$. The $y$-coordinates in each plot are uniform on $[0,1]$; a family fits well if the points fall roughly on the gray line.

We record a $p$-value of 0 when we cannot fit a distribution. The funds with $\left(\kappa_{3}, \kappa_{4}\right) \in \hat{R}$ have black points and the rest have gray. For comparison, we show the Anderson-Darling $p$-values if we use uncorrected Cornish-Fisher distributions (Figure 3(b)), defined at the end of Section 2, and normal distributions (Figure 3(c)). In Figure 3(b), we omit the fifty-nine cases where the uncorrected Cornish-Fisher expansion does not give a distribution function.

Both the Cornish-Fisher and uncorrected Cornish-Fisher fits have better-thanexpected $p$-values for many funds. We think this is largely because the four parameters allow the distribution to fit the data better than its population. We note that the correlation between many pairs of fund returns is too high for them to be plausibly independent.

Figures 4-6 show more detail for some of the fitted Cornish-Fisher distributions. Each chart shows a Q-Q plot, the fitted distribution function together with an empirical distribution function, and a histogram together with the fitted density function (solid) and a density function estimated by kernel density estimation. The $p$ shown is the $p$-value from the bootstrap Anderson-Darling test.

Depending on how accurate a fit is needed, twenty to thirty funds do not plausibly fit their (corrected) Cornish-Fisher distributions or do not have one. Most of these have one or two extreme values and $\left(\kappa_{3}, \kappa_{4}\right) \notin \hat{R}$, though ED12 (Figures 1(a) and 4(a)) does not. MS19 (Figure 4(b)) and HFFms42 (not shown) both have $\left(\kappa_{3}, \kappa_{4}\right) \notin \hat{R}$ but density functions that are likely not unimodal.

Figure 5 shows two better-fit distributions. LSeq55 (Figure 5(a)) is the 302nd best fit and SF64 (Figure 5(b)), the 149th. Figure 6 shows two weaker-fit distributions. GM11 (Figure 6(a)) is the 320th best fit. GM4 (Figure 6(b)) has the least good fit of the thirteen funds with negative kurtosis. We find that nine of these funds have Anderson-Darling-estimated $p$-values exceeding 0.9. To see why this might happen, consider the case $s=0$. The Jacobian has a zero at approximately $k=-0.139$, corresponding to a kurtosis of about -1.31 . And $\xi(u)$ is increasing between its roots at $\pm \sqrt{1-1 /(3 k)}$. Thus, in the worst case (when $k=-0.139$ ), the distribution is well defined for approximately 1.84 standard deviations on either side of the mean 
value. That is, only the extreme tails of the distribution function are undefined. These are not visible on the plot and do not affect the Anderson-Darling test.

The dashed lines in Figure 1 approximate and interpolate skewness and kurtosis corresponding to some of the zeros of the Jacobian. The lines are not simple. For example, we estimate three closely spaced zeros for skewness around \pm 2.7 . Nonetheless, we conjecture that the region featuring $R$ on which $G$ is invertible includes most of the points between $\hat{R}$ and this line. This would account for the very good fit of funds with kurtosis not in $\hat{R}$.

\subsection{CVaR estimation}

Section 3 showed that we can estimate quantiles and tail means using the CornishFisher distribution. The estimates should be less influenced by outliers than, for example, the method of Acerbi (2007) for estimating CVaR directly from the data.

We estimate CVaR at $\alpha=0.1$ in four different ways. These are as follows:

(i) from a piecewise-linear empirical distribution estimate (Acerbi 2007);

(ii) using the Cornish-Fisher distribution and (3.3);

(iii) using the uncorrected Cornish-Fisher distribution; and

(iv) using the Cornish-Fisher distribution together with shrinkage estimators to deal with estimation error.

Shrinkage estimators correct estimation error, which underestimates the smallest and overestimates the largest statistic in multivariate samples (Herold and Maurer 2006). We use the method of Jorion (1986) for the mean value and that of Ledoit and Wolf (2004) for the covariance matrix. The means are estimated as

$$
\overline{\boldsymbol{r}}^{*} \approx 0.234 \bar{r} \mathbf{1}_{n}+0.766 \overline{\boldsymbol{r}}
$$

where the usual estimate of the $n$ mean returns is $\overline{\boldsymbol{r}}, \mathbf{1}_{n}$ is a vector of $n 1 \mathrm{~s}$, and $\bar{r}$ is the mean of $\overline{\boldsymbol{r}}$. The covariance matrix estimate is

$$
S^{*} \approx 0.073 m I_{n}+0.927 S_{n}
$$

where $S_{n}$ is the unbiased estimator of the covariance matrix, $m \approx 22.81$ is the average variance of the $n=339$ funds and $I_{n}$ is the $n \times n$ identity matrix. Supplement $\mathrm{D}$ online shows the details of the calculation. We do not discuss specific choices of shrinkage estimators. We do, however, note that they shrink the mean and variance toward the overall mean, and the correlations toward zero. We expect them to increase small and reduce large values of CVaR. 
J. D. Lamb et al

FIGURE 4 Poor-fit Cornish-Fisher distributions: part (a).
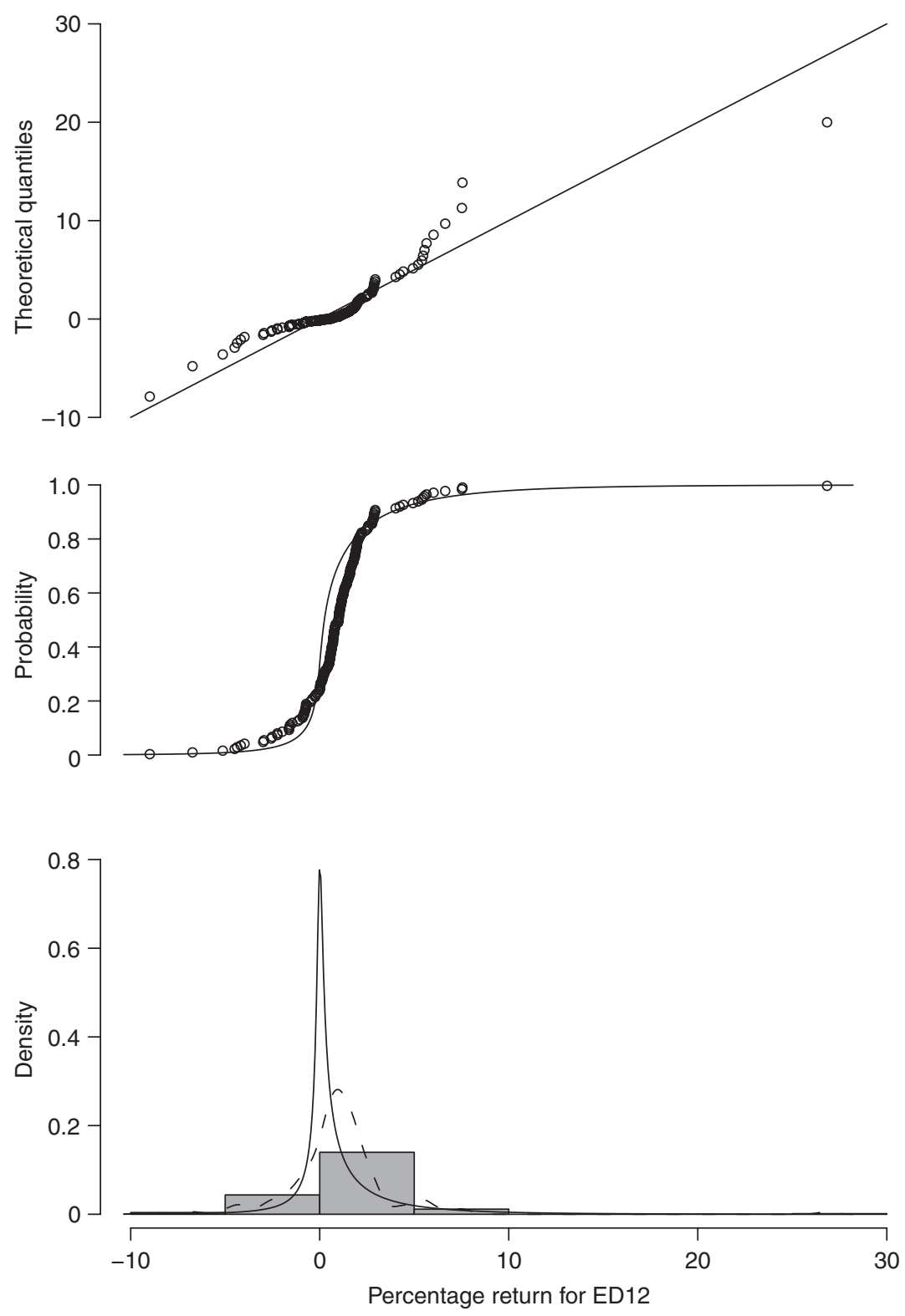

Mean: 1.026. Standard deviation: 3.12. Skewness: 3.429. Kurtosis: 30.33. $p=0.000$. 
FIGURE 4 Poor-fit Cornish-Fisher distributions: part (b).
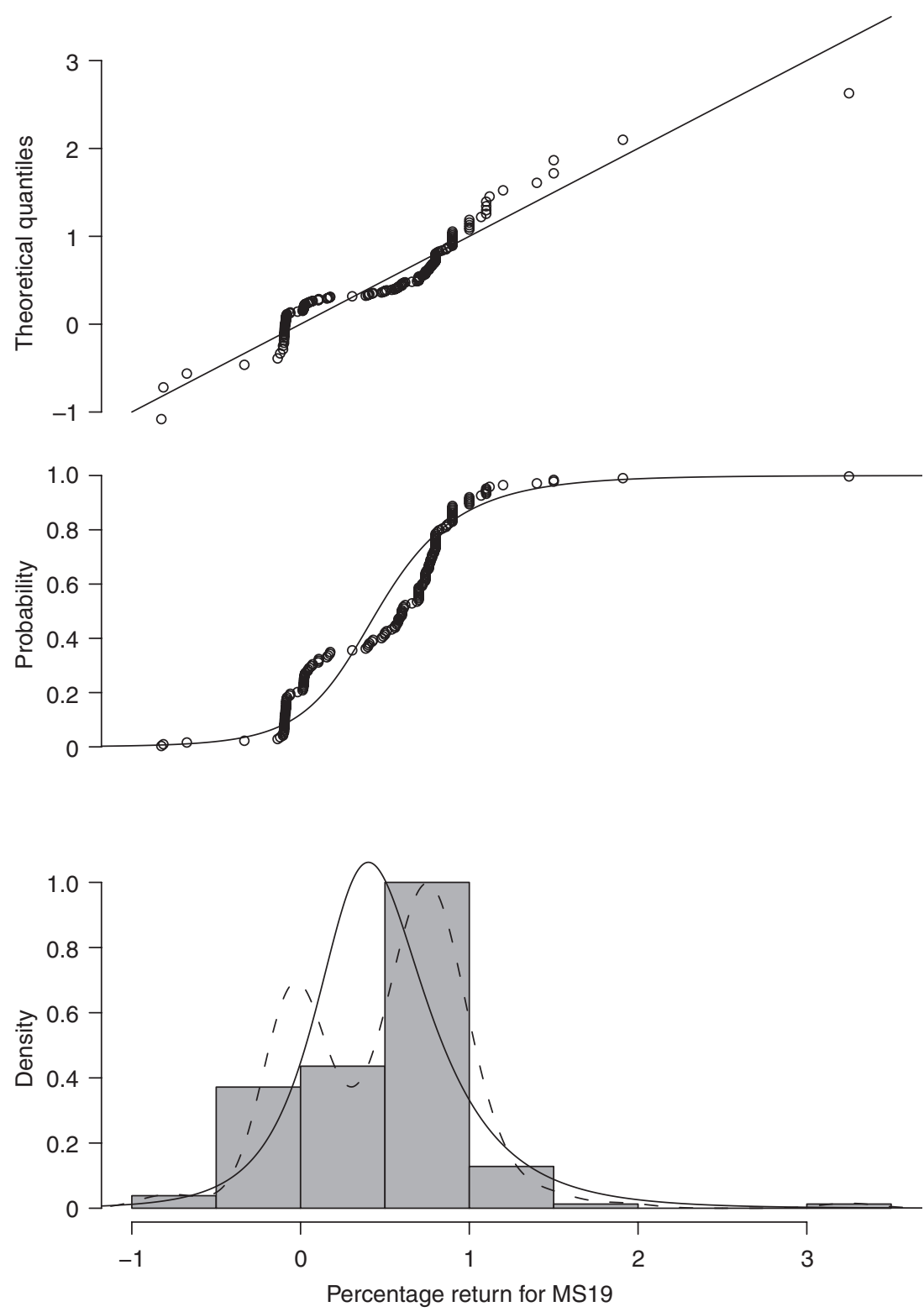

Mean: 0.497. Standard deviation: 0.515. Skewness: 0.762. Kurtosis: 4.407. $p=0.000$. 
FIGURE 5 Good-fit Cornish-Fisher distributions: part (a).
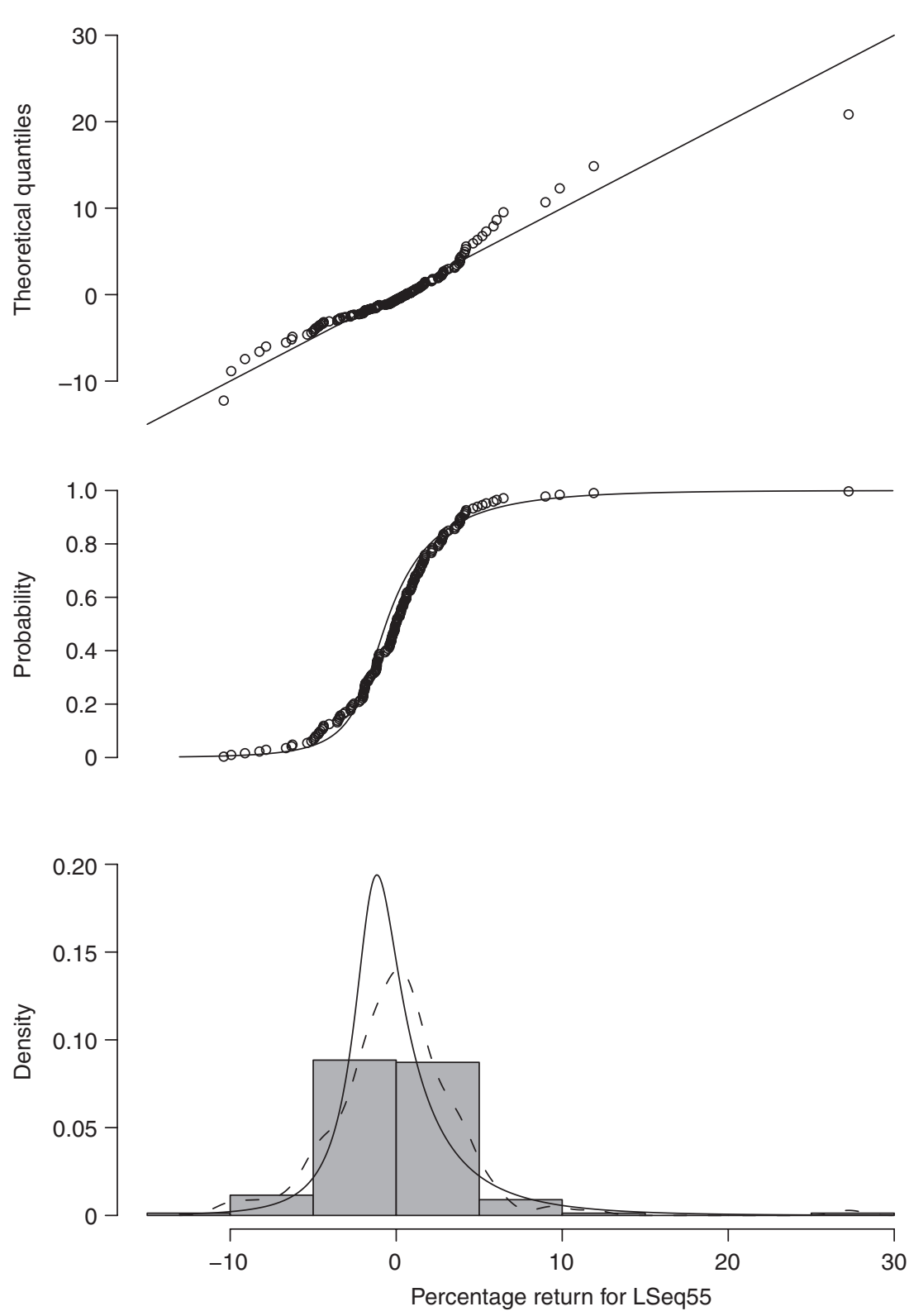

Mean: 0.042. Standard deviation: 4.094. Skewness: 1.816. Kurtosis: 12.313. $p=0.052$. 
FIGURE 5 Good-fit Cornish-Fisher distributions: part (b).
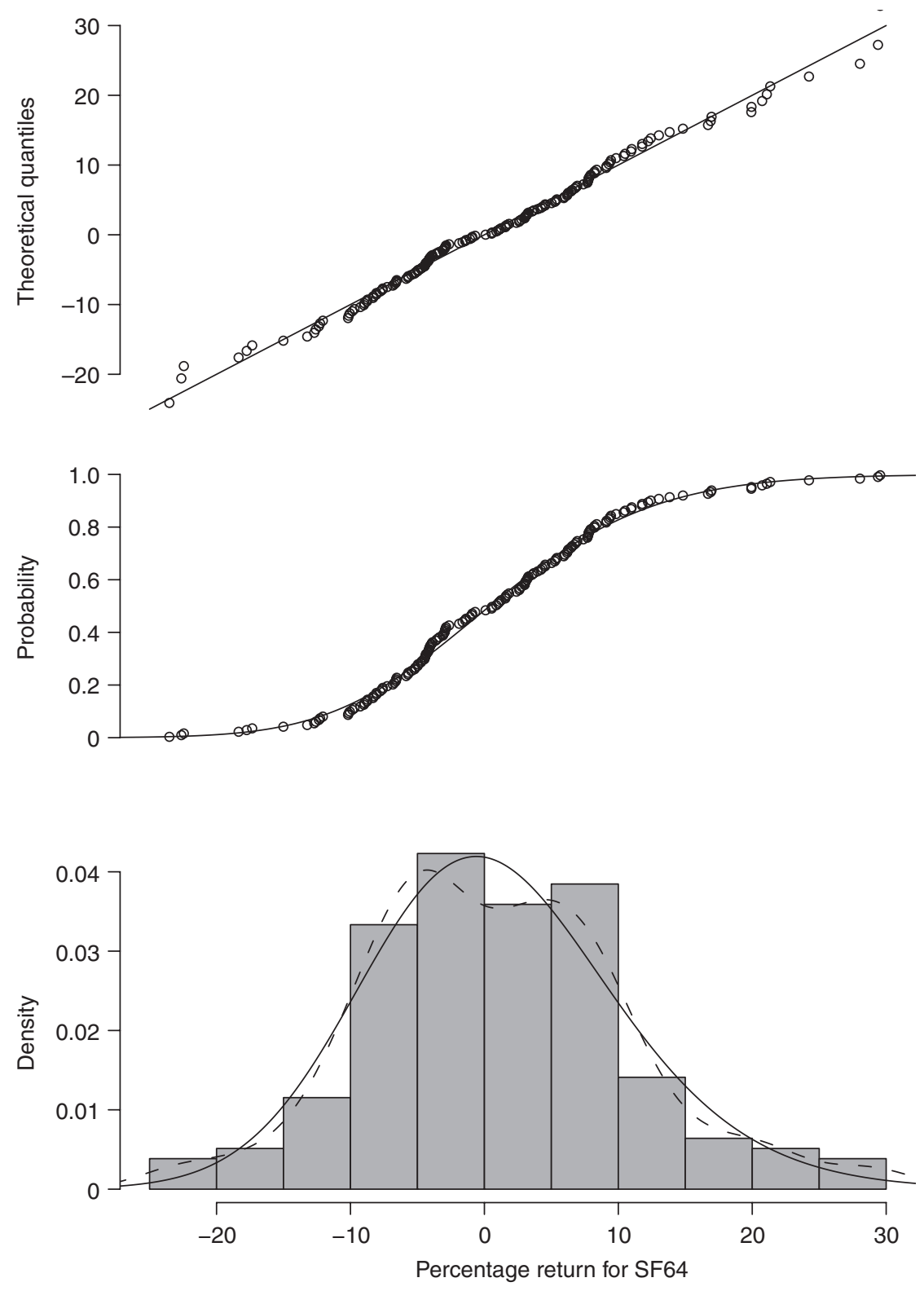

Mean: 0.931. Standard deviation: 9.954. Skewness: 0.344. Kurtosis: 0.47. $p=0.863$. 
FIGURE 6 Weak-fit Cornish-Fisher distributions: part (a).
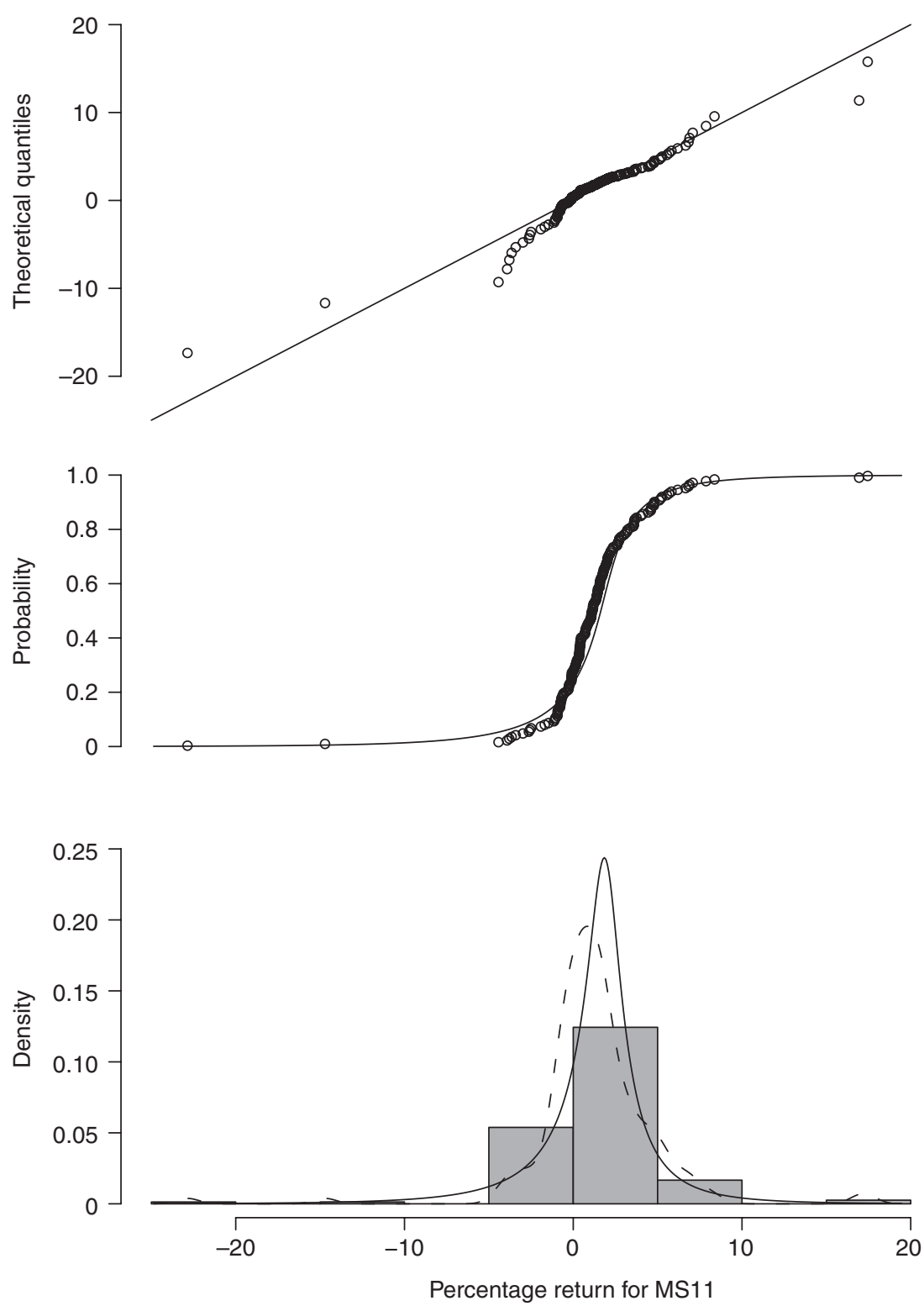

Mean: 1.309. Standard deviation: 3.773. Skewness: -1.115 . Kurtosis: $15.195 . p=0.014$. 
FIGURE 6 Weak-fit Cornish-Fisher distributions: part (b).
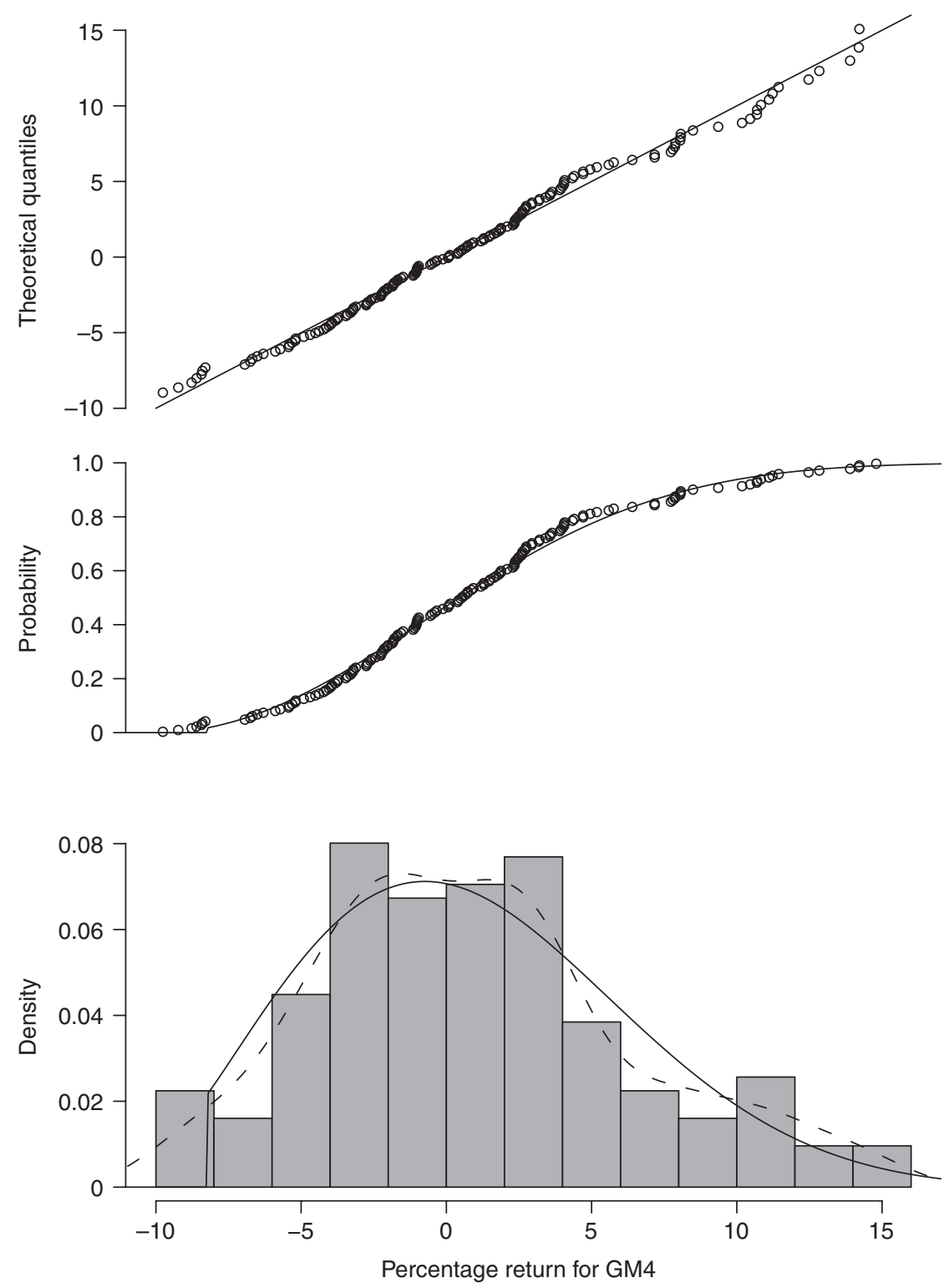

Mean: 0.956. Standard deviation: 5.412. Skewness: 0.489. Kurtosis: $-0.102 . p=0.049$. 
Figure 7 compares the various CVaR estimates for the 339 funds. Section 3 notes that we can sometimes estimate CVaR when $\left(\kappa_{3}, \kappa_{4}\right) \notin \hat{R}$. For example, for GM4 (see Figure 6(b)), $\xi$ is increasing between about -0.917 and 1.428 so that the Cornish-Fisher expansion defines a quantile function except in the extreme $(<0.005)$ tails. So, we compute CVaR in all 339 cases, including the seven in Section 4 where the fit fails. The circles show funds with skewness and kurtosis in $R$ (see Figure 1); the squares show funds with skewness and kurtosis in $\hat{R}$; and the triangles show the worst-behaved funds, including the seven where the fit fails. As expected, Cornish-Fisher CVaR fails in most of the seven worst cases. Figure 7(a) shows a good fit between the empirical CVaR and the Cornish-Fisher distributions. Except in extreme cases, we can attribute variations to the smoothing effect of the Cornish-Fisher distribution.

Figure 7(b) shows the problem of using the uncorrected Cornish-Fisher expansion: CVaR is often underestimated. This usually happens when $\left(\kappa_{3}, \kappa_{4}\right) \notin R$.

Figure 7(c) illustrates why we should consider estimation error; otherwise, we tend to underestimate CVaR when it is small and overestimate it when it is large. Note especially that the empirical CVaR estimate can be less than $50 \%$ of shrinkage CVaR or even negative when CVaR is small.

The shrinkage CVaR estimates here use shrinkage only for mean and variance. We expect (see Figure 2) better estimators of skewness and kurtosis to shrink the more extreme estimates of these values. Section 4.2 notes that the worst-fit funds tend to have extreme skewness and kurtosis and so are likely to fit better if we can find such estimators. In addition, we expect negative kurtosis to be reduced, leading to risk estimators that are valid even in the more extreme tails of the Cornish-Fisher distribution. Martellini and Ziemann (2010) discuss possible shrinkage estimators for the co-cumulants. These can be used in the univariate case, and we discuss them further in the next section.

\subsection{Multivariate fitting}

Section 3.2 describes how we can fit a multivariate Cornish-Fisher distribution using a mean vector, covariance matrix, skewness and kurtosis tensors. We cannot illustrate every example of multivariate portfolio fit and, in general, we expect randomly chosen portfolios to fit as well as the funds above. So, we illustrate using an example of a problematic portfolio and show how the Cornish-Fisher distribution can help with risk estimation.

The portfolio contains eleven of the 339 funds, with weights between 0 and 0.313 . Figure 8 shows the empirical distribution function as circles. We choose this portfolio because it has multiple returns slightly greater than -1 , giving an empirical estimate for VaR at $10 \%$ of 1 . This likely underestimates the risk of future portfolio returns. 
FIGURE 7 Comparison of CVaR estimates.

(a)

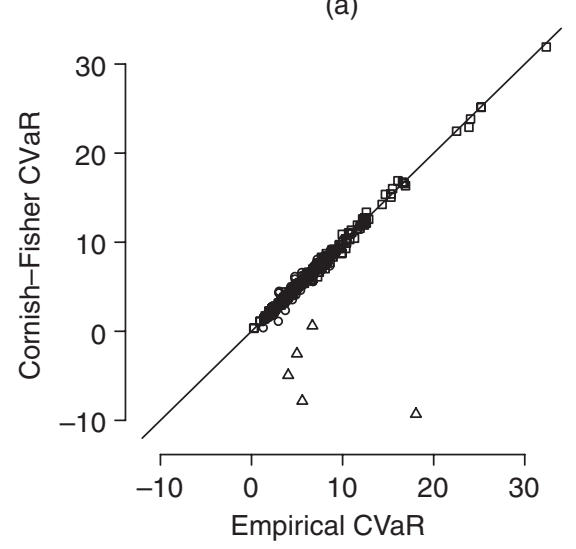

(b)

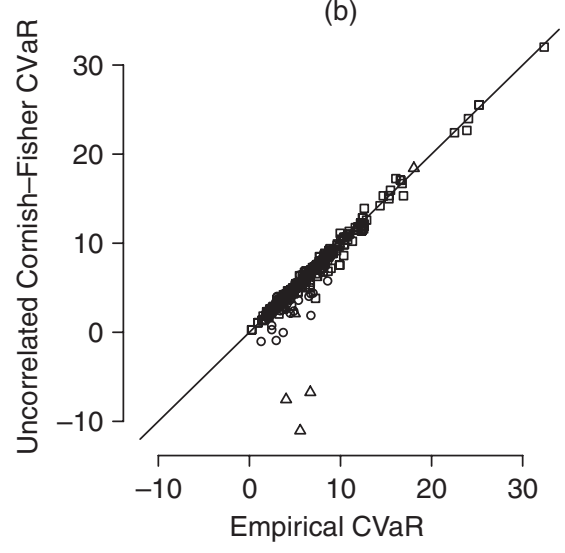

(c)

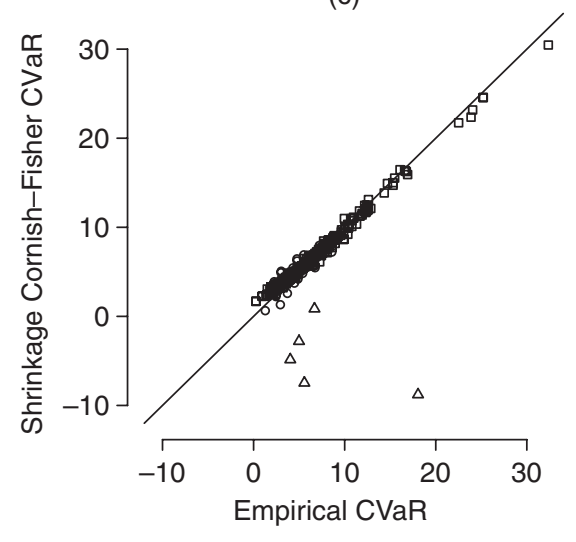

Figure 8(a) shows the (corrected) Cornish-Fisher distribution fitted to the empirical data (that is, using data estimates for means, covariances, co-skewnesses and co-kurtoses). The bootstrap Anderson-Darling $p$-value confirms an obviously poor fit. In this case, the Cornish-Fisher fit gives us a worse estimate of VaR but tells us that the empirical estimate is also likely unreliable.

Figure 8(b) shows the (corrected) Cornish-Fisher distribution fitted to the data, with the most extreme left- and right-tail values removed. Note the substantial changes in skewness and kurtosis and the much more plausible fit. This time, the Cornish-Fisher distribution gives us a more conservative and plausible estimate of VaR than the empirical estimate. 
FIGURE 8 Fitting a portfolio from the multivariate distribution.

(a)

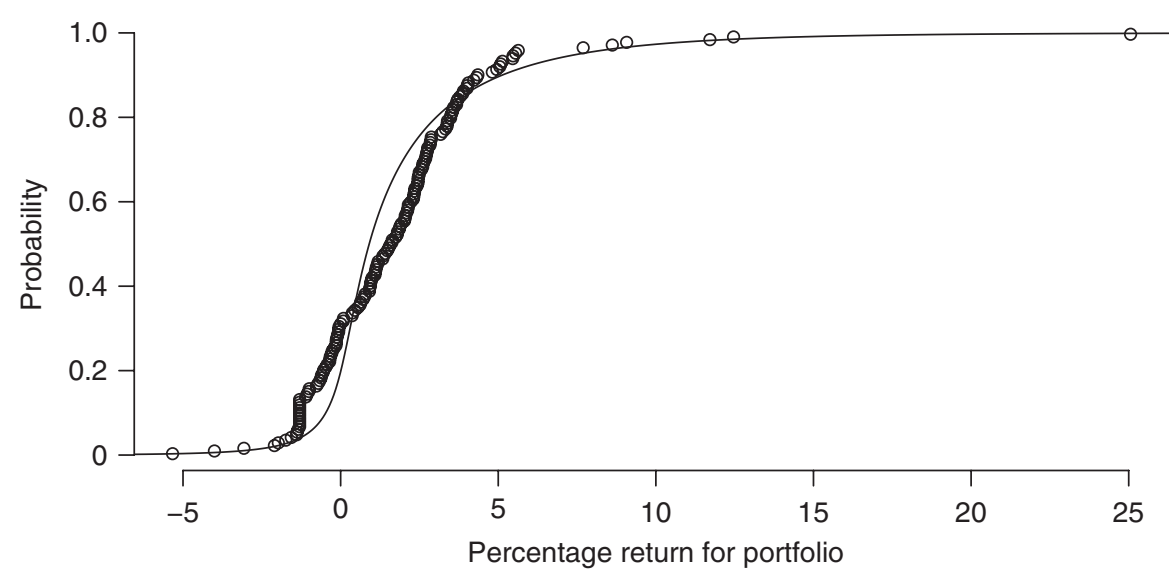

(b)

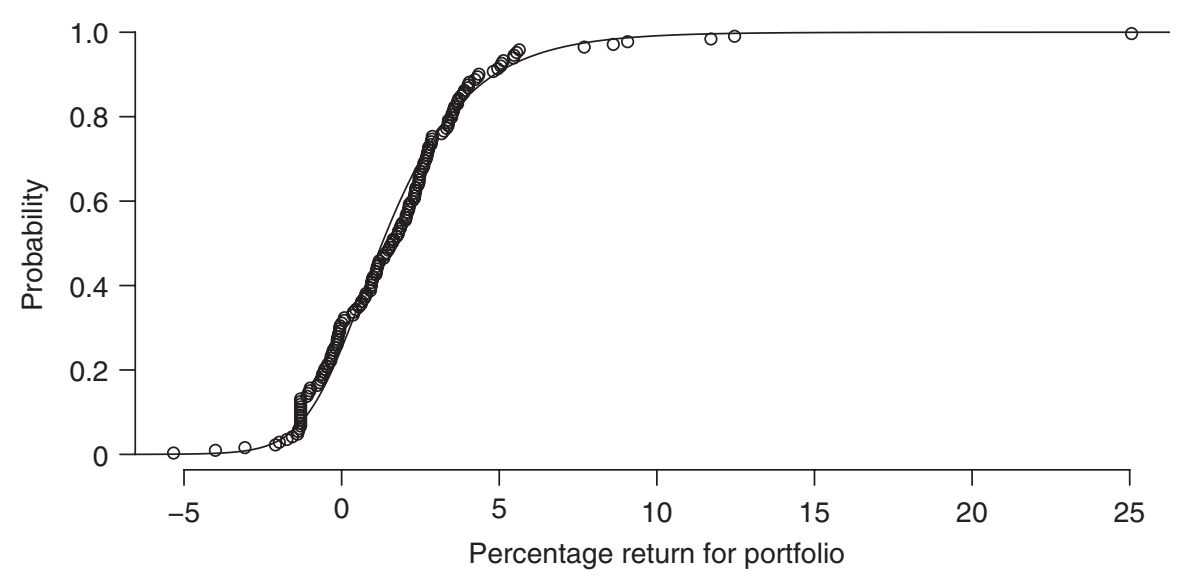

(a) Mean: 1.771. Standard deviation: 3.176. Skewness: 3.001. Kurtosis: 18.819. $p=0.001$. (b) Mean: 1.666. Standard deviation: 2.517 . Skewness: 1.142 . Kurtosis: $3.105 . p=0.301$.

If one's aim is to estimate risk in individual cases, then censoring extreme data points may be sufficient and will, for example, improve the fits in Figures 4 and 6. More generally, it is likely that co-cumulant shrinkage estimators such as those of Martellini and Ziemann (2010) could be combined with the multivariate CornishFisher distribution to give good risk estimators for most portfolios. 


\section{CONCLUSION}

Extending and correcting an idea of Maillard (2012), we demonstrate how to fit a Cornish-Fisher distribution with specified mean, variance, skewness and kurtosis. We show that the distribution has desirable properties such as unimodality and normal-like tails. We also demonstrate that we can fit the distribution for a range of skewnesses and kurtoses big enough to fit a multivariate Cornish-Fisher distribution by excluding only the rarest of asset returns.

Risk measures such as CVaR and VaR are based on the tail of a distribution. This makes estimates from data very sensitive to extremes. Estimating from our Cornish-Fisher distribution has advantages. First, the distribution shape - flat-tailed, smooth and unimodal - is common in asset returns. Second, we can use it alongside other methods to reduce estimation risk. This makes it more promising as we develop improved shrinkage estimators. In the univariate case, we suggest using the Cornish-Fisher distribution to help identify where estimating CVaR or VaR may be problematic. In the multivariate case, we recommend using shrinkage estimators to reduce estimation error in estimating risk. The Cornish-Fisher distribution allows us to do this for CVaR and VaR and is particularly important in helping us avoid underestimating risk in lower-risk assets.

Proposition 3.2 tells us that risk measures like CVaR will be twice-continuously differentiable as a function of the coefficients of the linear combination. This makes minimizing risk over a set of assets easier, especially when we use risk measures such as CVaR that are convex functions of the coefficients of a linear combination of assets.

Scope and space have limited our exploration of two issues. First, we have ignored time series effects to simplify Section 4. We can include these effects in principle (Gabrielsen et al 2015), but it would be useful to see what happens in practice.

Second, we have limited our discussion of estimation error and shrinkage estimators to some well-known methods for mean, variance and covariance (Herold and Maurer 2006; Ledoit and Wolf 2004). This area could be further explored. One benefit of the Cornish-Fisher distribution is that it allows the possibility of developing shrinkage estimators for skewness and kurtosis. Just as Ledoit and Wolf (2004) shrink not just the variances but the whole covariance matrix, such methods could shrink the co-skewess and co-kurtosis tensors. The Cornish-Fisher distribution is then likely to be useful: it is more likely to fit. Moreover, while we can change the mean and variance of data without affecting any other cumulants, changing the covariance is problematic, and we know of no way to change its skewness or kurtosis without affecting other cumulants. Shrinking skewness and kurtosis would prevent us from estimating risk measures such as VaR and CVaR empirically; however, the Cornish-Fisher distribution estimates would remain. 


\section{SUPPLEMENTARY MATERIAL}

\section{Making Cornish-Fisher distributions fit: detailed derivations}

The file CornishFisherSupplement.pdf, available online, provides detailed derivations of some of the results used in this paper (portable document file format).

\section{R-package for Cornish-Fisher functions}

The file CornishFisher 1.0.tar.gz, also available online, contains code to compute the Cornish-Fisher expansions, distribution functions, quantile functions and bootstrap Anderson-Darling test described in this paper (gnu zipped tar file format).

\section{DECLARATION OF INTEREST}

The authors report no conflicts of interest. The authors alone are responsible for the content and writing of the paper.

\section{REFERENCES}

Acerbi, C. (2002). Spectral measures of risk: a coherent representation of subjective risk aversion. Journal of Banking \& Finance 26(7), 1505-1518 (https://doi.org/10.1016/ S0378-4266(02)00281-9).

Acerbi, C. (2007). Coherent measures of risk in everyday market practice. Quantitative Finance 7(4), 359-364 (https://doi.org/10.1080/14697680701461590).

Bali, T. G., Gokcan, S., and Liang, B. (2007). Value at risk and the cross-section of hedge fund returns. Journal of Banking \& Finance 31(4), 1135-1166 (https://doi.org/10.1016/ j.jbankfin.2006.10.005).

Cheng, R. C. H. (2006). Validating and comparing simulation models using resampling. Journal of Simulation 1(1), 53-63 (https://doi.org/10.1057/palgrave.jos.4250009).

Cornish, E. A., and Fisher, R. A. (1938). Moments and cumulants in the specification of distributions. Revue de l'Institut International de Statistique 5, 307-322 (https://doi.org/ 10.2307/1400905).

Gabrielsen, A., Kirchner, A., Liu, Z., and Zagalia, P. (2015). Forecasting value-at-risk with time-varying variance, skewness and kurtosis in an exponential weighted moving average framework. Annals of Financial Economics 10, 1-29 (https://doi.org/10.1142/S2010 495215500050).

Herold, U., and Maurer, R. (2006). Portfolio choice and estimation risk: a comparison of Bayesian to heuristic approaches. ASTIN Bulletin 36(1), 135-160 (https://doi.org/ 10.1017/S0515036100014434).

Hill, G. W., and Davis, A. W. (1968). Generalized asymptotic expansions of Cornish-Fisher type. Annals of Mathematical Statistics 39(4), 1264-1273 (https://doi.org/10.1214/ aoms/1177698251).

Hitaj, A., Martellini, L., and Zambruno, G. (2012). Optimal hedge fund allocation with improved estimates for coskewness and cokurtosis parameters. Journal of Alternative Investments 14(3), 6-16 (https://doi.org/10.3905/jai.2012.14.3.006). 
Jondeau, E., Jurczenko, E., and Rockinger, M. (2017). Moment component analysis: an illustration with international stock markets. Journal of Business and Economic Statistics 36(4), 576-598 (https://doi.org/10.1080/07350015.2016.1216851).

Jorion, P. (1986). Bayes-Stein estimation for portfolio analysis. Journal of Financial and Quantitative Analysis 21(3), 279-292 (https://doi.org/10.2307/2331042).

Ledoit, O., and Wolf, M. (2004). A well-conditioned estimator for large-dimensional covariance matrices. Journal of Multivariate Analysis 88(2), 365-411 (https://doi.org/10.1016/ S0047-259X(03)00096-4).

Liang, B., and Park, H. (2007). Risk measures for hedge funds: a cross-sectional approach. European Financial Management 13(2), 333-370 (https://doi.org/10.1111/ j.1468-036X.2006.00357.x).

Maillard, D. (2012). A user's guide to the Cornish Fisher expansion. Working Paper, Social Science Research Network (https://doi.org/10.2139/ssrn.1997178).

Maillard, D. (2013). More on Cornish-Fisher: distribution density and boundary conditions. Working Paper, Social Science Research Network (https://doi.org/10.2139/ssrn .2236338).

Martellini, L., and Ziemann, V. (2010). Improved estimates of higher-order comoments and implications for portfolio selection. Review of Financial Studies 23(4), 1467-1502 (https://doi.org/10.1093/rfs/hhp099).

Moreno, D., and Rodríguez, R. (2009). The value of coskewness in mutual fund performance evaluation. Journal of Banking \& Finance 33(9), 1664-1676 (https://doi.org/ 10.1016/j.jbankfin.2009.03.015).

Morningstar (2014). Morningstar: independent investment research. Website. URL: www .morningstar.com.

Rigby, R. A., and Stasinopoulos, D. M. (2004). Smooth centile curves for skew and kurtotic data modelled using the Box-Cox power exponential distribution. Statistics in Medicine 23, 3053-3076 (https://doi.org/10.1002/sim.1861).

Tee, K.-H. (2009). The effect of downside risk reduction on UK equity portfolios included with managed futures funds. International Review of Financial Analysis 18(5), 303-310 (https://doi.org/10.1016/j.irfa.2009.09.007). 
